\title{
Orientação acadêmica e profissional dos estudantes com deficiência nas universidades italianas
}

\author{
Leonardo Santos Amâncio Cabral ${ }^{1}$ \\ Enicéia Gonçalves Mendes² \\ Lucia de Anna ${ }^{3}$
}

\section{Introdução}

No decênio 2005-2014, a Unesco indicou a necessidade de se adotar novos comportamentos e práticas para o desenvolvimento sustentável de uma sociedade, destacando a educação como promotor central desse processo (UNESCO, 2009). Todavia, ainda segundo a Unesco, a educação das pessoas com deficiência e sua plena inclusão na sociedade continua a ser um dos mais desafiadores objetivos do século XXI.

O "Plano de ação do Conselho da Europa 2006-2015 para a promoção dos direitos e da plena participação das pessoas com deficiência na sociedade: melhorar a qualidade de vida das pessoas com deficiência na Europa" (CONSIGLIO D’EUROPA, 2006) colocou em evidência não só a necessidade do acesso e permanência do público-alvo da educação especial na rede regular de ensino, mas também a da promoção de programas de transição eficientes e eficazes às pessoas com deficiência, em nível educacional e profissional, promovendo sua efetiva inclusão nas escolas, nas universidades e no mundo do trabalho.

1 UFSCar - Universidade Federal de São Carlos. Programa de Pós-Graduação em Educação Especial. São Carlos - São Paulo - Brasil. prof.leonardocabral@gmail.com

2 UFSCar - Universidade Federal de São Carlos. Programa de Pós-Graduação em Educação Especial. São Carlos - São Paulo - Brasil. egmendes@ufscar.br

3 Uniroma4 - Università degli Studi di Roma "Foro Italico". Laboratorio di Pedagogia Speciale. Roma - Roma - Itália. lucia.deanna@uniroma4.it 
Na Itália, particularmente, as iniciativas nessa direção começaram a partir da década de 1970, estimulada pela "Declaração dos Direitos das Pessoas com Deficiência” (ONU, 1975, art.3), que preconizava:

as pessoas com deficiência, qualquer que sejam a origem, natureza e gravidade de suas deficiências, têm os mesmos direitos fundamentais que seus concidadãos da mesma idade, o que implica, antes de tudo, o direito a uma vida decente, normal e plena na medida do possível.

Foi naquele período que o governo italiano determinou, por meio da Lei n. 517 de 1977, a matrícula compulsória de todas as crianças em idade escolar na rede regular de ensino (CABRAL, 2010, 2013; DE ANNA, 2014).

Como consequência dessa agenda nos debates nacionais e internacionais sobre as questôes relacionadas à inclusão das pessoas com deficiência, aumentou o fluxo das matrículas desse público nos contextos educacionais, de modo que, nos anos 1980, tal público foi chegando às universidades. A literatura científica dessa época, em vários países, apontava uma tendência política ao desenvolvimento de programas de suporte às pessoas com deficiência, voltados à transição da escola à universidade e/ ou ao mundo do trabalho (BROWN; KAYSER, 1981; BROLIN; ELLIOTT, 1984).

Na Itália, De Anna (1989, p.3) expunha a preocupação da sociedade e associaçóes em identificar meios de garantir às pessoas com deficiência "a sua continuidade educativa rumo aos níveis mais elevados de educação", pois nem todos conseguiam adquirir efetivamente o diploma de conclusão de curso. Os estudantes com deficiência psíquica, por exemplo, seguiam ainda um percurso alternativo que frequentemente os conduzia a um mero atestado de frequência. As pessoas com deficiência física e sensorial, por outro lado, ainda que timidamente, tendiam a concluir o segundo grau e a inscrever-se cada vez mais nas universidades, ainda que enfrentassem grandes dificuldades durante seu percurso acadêmico (DE ANNA, 1996).

Diante a tal fenômeno, a década de 1990 foi marcada pela necessidade de se implementar e aprimorar serviços de orientação acadêmica e profissional aos estudantes universitários com deficiência, como apontavam Norlander, Shaw e McGuire (1990); Rusch, Kohler e Rubin (1994) e Raskind e Higgins (1998).

Nesse sentido, a Lei italiana n. 390 de 1991, "Norme sul diritto agli studi Universitari", previu disposiçóes particulares para a promoção do acesso aos estudantes com deficiência nas universidades e, com a demanda cada vez mais crescente, a Legge-quadro per l'assistenza, l'integrazione sociale e i diritti delle persone handicappate, n. 104/1992, definiu critérios para a disposição de equipamentos técnicos, subsídios didáticos e recursos humanos, bem como de programas de intervenção segundo as necessidades de cada estudante, inclusive para sua transição ao mundo do trabalho. Naquele contexto, De Anna (1996, p.2) expunha: 
[... finalmente foi reconhecido que a pessoa com deficiência também é um recurso econômico a partir do momento em que for auxiliada a superar as diversas dificuldades advindas da situação de impedimento e a inserir-se plenamente na sociedade. A sua formação, desse modo, constitui-se em um elemento importante para o alcance de tal objetivo.

Frente à demanda advinda de tais iniciativas ao longo dos anos 1990, a lei n. 17, de 28 de janeiro de 1999, determinou que cada universidade nominasse um pró-reitor para as questóes relacionadas à deficiência, o qual deveria desenvolver as funçóes de coordenação, monitoramento e suporte às iniciativas necessárias à inclusão dos estudantes com deficiência no âmbito da própria universidade.

Diante ao desafio imposto, os pró-reitores para as questôes relacionadas à deficiência reuniram-se em diversas ocasióes decidindo, em 2001, constituírem a Conferenza Nazionale Universitaria dei Delegati per la Disabilità (CNUDD). Com a ativa colaboração da Conferenza dei Rettori delle Università Italiane (Crui), a CNUDD teve como base princípios da participação ativa, do acolhimento e sensibilização, da autonomia, da igualdade de oportunidades, os quais permearam o processo de implementação da normativa n. 17/1999.

Naquele mesmo ano, foi aprovada a "Declaração Internacional de Montreal sobre Inclusão", a qual determinou aos governos, empregadores, trabalhadores e à sociedade civil a se comprometerem com a promoção da inclusão dos grupos historicamente segregados, particularmente o grupo constituído pelas pessoas com deficiência, em todos os ambientes, produtos e serviços (CANADÁ, 2001). Todavia, autores como Collins e O’Mahony (2001) e Konur (2002) relevavam a existência de numerosos obstáculos em aspectos estruturais, pedagógicos, culturais e sociais que ainda deveriam ser superados, particularmente no que se referia aos contextos universitário e do mundo do trabalho.

Frente a isso, a "Convenção Internacional sobre os Direitos das Pessoas com Deficiência”, de 2006, reforçou princípios e metas que permeariam a superação de tais obstáculos. No que se refere à universidade e ao mundo do trabalho, após a Convenção, houve um considerável aumento do número de serviços voltados à orientação acadêmica e profissional, motivando os estudantes universitários com deficiência a investirem em sua formação e a colocar em prática sua profissionalização (LANDMARK; JU; ZHANG, 2010; REPETTO et al., 2011; CARTER; AUSTIN; TRAINOR, 2012).

Pode-se afirmar, portanto, que o segundo decênio do século XXI traz consigo a herança histórica de princípios, perspectivas culturais, políticas, sociais, econômicas e ideológicas que, atualmente, contribuem para a promoção da plena participação das pessoas com deficiência no contexto escolar, na sua transiçáo da escola para a universidade e da universidade para o mundo do trabalho 
(DE ANNA; 1996, 2002; MENDES, 2006; EBERSOLD, 2001, 2003, 2008a; 2008b; MOLITERNI; DI STASIO; CARBONI, 2011; LASCIOLI, 2012).

Todavia, questiona-se: como as universidades vêm sendo organizadas para que se enfrente esse processo com eficácia? Quais estratégias atuais têm sido direcionadas para a orientação acadêmica e profissional do público-alvo da educação especial nas universidades? Diante de tais questionamentos, parece necessário explorar, aprofundar e organizar um quadro de boas práticas que possa responder às necessidades e valorizar as competências e habilidades das pessoas com deficiência, com vistas a propor um efetivo programa de formação profissional que responda às exigências de um contexto socioeconômico globalizado (HOGSTEDT; WEGMAN; KJELLSTROM, 2007; MACLEAN; LAI, 2011).

Nesta direção, a presente pesquisa teve como objetivo principal identificar, reunir, analisar e evidenciar, particularmente no que se refere ao contexto italiano, as possíveis boas práticas de orientação acadêmica e profissional dos estudantes universitários com deficiência.

\section{A organização das universidades italianas}

O sistema universitário italiano é representado por 96 instituições de diversas tipologias: estatais, não estatais, institutos especiais, universidade para estrangeiros, escolas superiores, institutos de alta formação doutoral e telemáticas. A organização de cada universidade vem representada pelo organograma a seguir:

Organograma das universidades/ateneus italianos

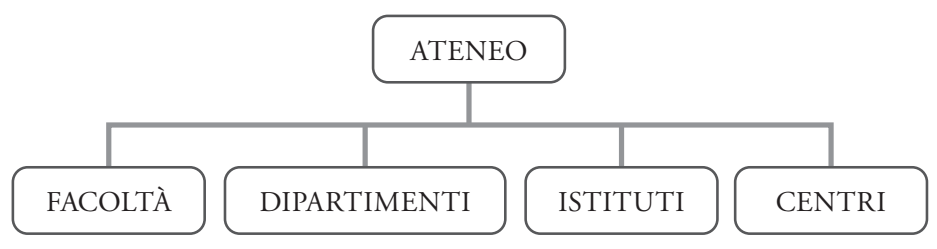

Fonte: Cabral (2013).

Segundo os dados do Miur (2012), dos 96 ateneus italianos, 68 são estatais, e foi neste tipo de instituição que o estudo foi realizado. De modo geral, 35,8\% dos ateneus estatais são distribuídos no Norte da Itália, 34,4\% no Centro e 29,8\% no Sul, como representa o Mapa e a Tabela 1 a seguir: 
Mapa de distribuição dos ateneus estatais sobre o território italiano

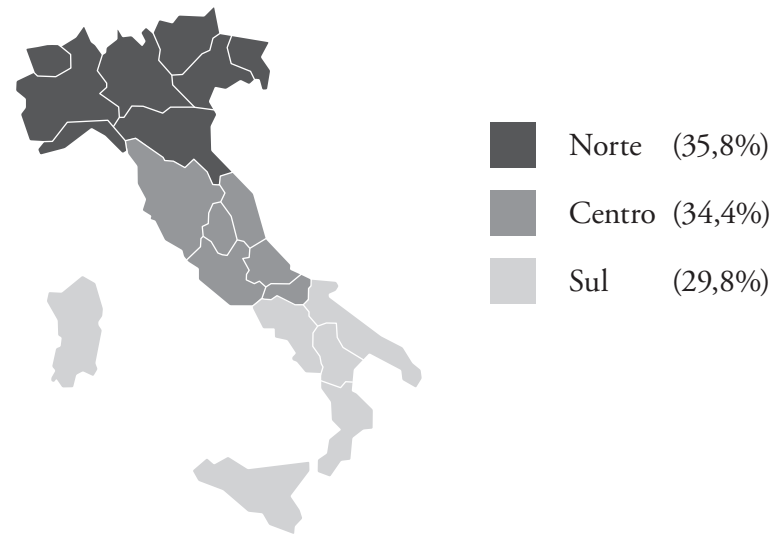

Fonte: Cabral (2013).

Tabela 1: Distribuição dos ateneus estatais na Itália, segundo a região

\begin{tabular}{|c|c|c|c|c|c|}
\hline \multicolumn{6}{|c|}{ Número de Universidades Estatais na Itália } \\
\hline Norte & \multicolumn{2}{|c|}{ Centro } & Sul \\
\hline Trentino-Alto Adige & 1 & Toscana & 8 & Molise & 1 \\
\hline Piemonte & 3 & Marche & 4 & Campania & 6 \\
\hline Lombardia & 8 & Umbria & 2 & Puglia & 4 \\
\hline Vêneto & 4 & Lazio & 6 & Basilicata & 1 \\
\hline Friuli-Veneza Giulia & 3 & Abruzzo & 3 & Calábria & 3 \\
\hline Liguria & 1 & - & - & Sicília & 3 \\
\hline Emilia-Romagna & 4 & - & - & Sardenha & 2 \\
\hline Total & $\mathbf{2 4}$ & Total & $\mathbf{2 3}$ & Total & $\mathbf{2 0}$ \\
\hline
\end{tabular}

Fonte: Miur (2012)

\section{Os estudantes com deficiência nas universidades italianas}

Foi após a Lei 17/1999 que se observa um crescimento exponencial do número de estudantes com deficiência matriculados, de 5.514 para 15.994 no ano acadêmico 2009/2010 (MIUR, 2012), representando 5,4\% do número total de 297.441 estudantes matriculados em todo o território nacional (MIUR, 2012). É importante ressaltar que este aumento ocorreu em um contexto no qual o número global dos alunos matriculados diminuiu no período correspondente, conforme indica o Istituto Nazionale di Statistica. 
Particularmente na Università degli Studi di Roma "Foro Italico" (Uniroma4), na Università degli Studi di Roma Tre (Uniroma3) e na Università degli Studi di Messina (UniMe), que constituíram a amostra do presente estudo, esse aumento também pôde ser observado:

Gráfico 1: Evolução do número de estudantes com deficiência entre o ano letivo 2005/2006 e 2009/2010 nos ateneus Uniroma3, Uniroma4 e UniMe

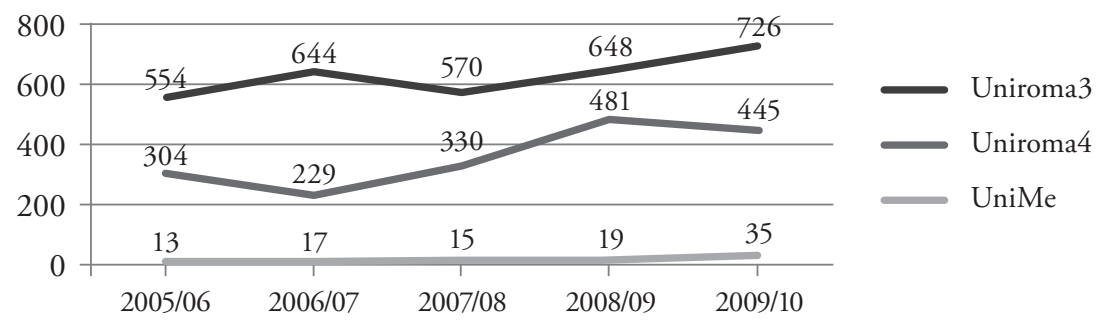

Fonte: Cabral (2013).

Na Itália, o serviço de Tutorato Specializzato agli studenti com disabilità e disturbi specifici dell'apprendimento é a estrutura responsável pelo acolhimento e o apoio durante todo o percurso universitário do estudante, inclusive o momento de sua transição à vida adulta e ao mundo do trabalho. De Anna (1996) destaca que o acolhimento é o momento mais delicado para um estudante, principalmente no caso em que a pessoa tem necessidades especiais e impedimentos.

Todavia, isso não quer dizer que o acolhimento e a orientação desta população sejam responsabilidades atribuídas a uma única estrutura universitária, pois promover a inclusão em todo o contexto universitário envolve esforço, colaboração e sinergia entre as diversas estruturas da universidade, estendendo-se também às varias estruturas do território, segundo as Leis 104/1992 e 17/1999 e as Diretrizes da CNUDD.

Quanto à questão do acesso ao mercado de trabalho e orientação profissional das pessoas com deficiência, a literatura indica que esta surgiu no contexto italiano no final dos anos 1980, ainda que, segundo De Anna (1989), as iniciativas fossem realizadas de modo superficial e generalista.

É a partir da década de 1990 que podemos identificar e colocar em evidência algumas normativas nacionais destinadas a propor e a colocar em prática estratégias orientadas à garantia do acesso das pessoas com deficiência ao mundo do trabalho. Destaca-se, particularmente, a Lei n. 68, de 12 de março de 1999, a qual predispôs diretivas para uma melhor organização em âmbito educacional, estrutural e de gestão, inclusive ao membro interno da universidade, a fim de consentir aos estudantes com deficiência a efetiva realização do seu projeto de vida profissional. 
As disposiçóes dessa lei foram reforçadas pelas Diretrizes da CNUDD, as quais dedicaram um capítulo inteiro sobre a orientação profissional dos estudantes universitários com deficiência bem como na sessão Lavoro e Occupazione, da Convenção sobre os Direitos das Pessoas com Deficiência (ONU, 2006), ratificada pela Lei italiana n. 18, de 3 de março de 2009.

\section{Metodologia}

O presente estudo compôs a agenda do projeto "Developing a support methodology of disabled students allowing to conjugate effectively academic success and access to employment", financiado pelo Programa Europeu Leonardo da Vinci em Vocational Education and Training.

A metodologia deste subprojeto envolveu um estudo de caso exploratório-descritivo, o qual abrangeu uma proposta de orientação acadêmica e profissional a 20 estudantes universitários com deficiência, todos em fase de transição universidade - mundo do trabalho. Dos 20 estudantes universitários com deficiência, metade tinha deficiência física, 30\% deficiência auditiva, 10\% deficiência visual, $5 \%$ deficiência sensorial múltipla (visual e auditiva) e 5\% distúrbios de origem médica (transplante de coração).

Os estudantes participantes eram provenientes da Università degli Studi di Roma "Foro Italico" (10 estudantes); Università degli Studi di Roma Tre (8 estudantes); e Università degli Studi di Messina (2 estudantes), sendo 50\% do curso de Educação Física, 20\% das Ciências da Educação, 5\% de Letras, 15\% de Cinema, 5\% das Ciências Políticas e 5\% da Economia.

Além dos 20 estudantes universitários com deficiência, participaram do estudo 3 tutores acadêmicos e 20 tutores empresariais de ONGs, instituições públicas e privadas.

O estudo envolveu a implementação e avaliaçáo de um programa de orientação acadêmica e profissional aos estudantes universitários com deficiência participantes, para o contexto das três universidades italianas mencionadas, tendo como base um programa semelhante dinamarquês. ${ }^{4}$

$4 \mathrm{O}$ modelo dinamarquês, referência para o projeto, foi representado por práticas e instrumentos utilizados pela Universidade de Aarhus. Vale ressaltar que o mesmo foi implementado nos contextos da França, da Itália, da Dinamarca e da Irlanda, questionado, discutido em reunióes periódicas e adaptado em cada contexto. Para conhecer mais sobre o projeto mais amplo, acesse: www.universemploi.inshea.fr. 


\section{Resultados}

Os resultados aqui relatados foram organizados em dois temas, sendo que o primeiro descreve o funcionamento do programa e a sinergia entre os serviços. Em um segundo momento são apresentadas as programas de acolhimento e orientação.

\section{O funcionamento do programa e a sinergia entre os serviços}

As diretrizes nacionais de orientação acadêmica e profissional dos estudantes universitários com deficiência destacam a importância da colaboração entre os serviços de tutoria especializada das universidades e aqueles presentes no território municipal, provincial, regional ou nacional.

Estão disponíveis, para tanto, serviços fornecidos pelas Unidades Locais de Saúde (USL), articulados com associaçôes como o Servizio Integrazione Lavorativa (SIL), a Unità Operativa per l'Inserimento Lavorativo (Uoil), as agências de mediação com o mundo do trabalho e as associações de pessoas com deficiência. Preconiza-se, sobretudo, a cooperação entre os atores envolvidos nesse processo (universidade, serviços, familiares e estudantes), para que se promova efetivamente a transição das pessoas com deficiência ao mercado de trabalho.

Particularmente, no que se refere à orientação profissional dos estudantes universitários com deficiência, é prevista a colaboração entre os Serviços de Estágios e Colocação no Mercado de Trabalho e o de Tutoria Especializada da Universidade, a fim de facilitar o encontro entre a demanda e a oferta de trabalho, fornecendo, inclusive, orientação no momento da escolha e durante a formação profissional, principalmente por meio de estágios. $\mathrm{O}$ excerto da entrevista descrito a seguir, de um dos tutores entrevistados, ilustra o funcionamento dessa tutoria:

A Tutoria Especializada tem um banco de dados no qual são inseridas todas as informaçôes sobre os alunos, principalmente aquelas que consideramos importante para a orientaçấo durante sua carreira acadêmica, desde o tipo de deficiência até os seus conhecimentos e necessidades educacionais especiais. Em caráter complementar, o Serviço de Estágios e Colocação no Mercado de Trabalho reúne informações sobre suas habilidades e objetivos profissionais, a fim de poder realizar a devida correspondência com a demanda apresentada pelo mundo do trabalho. (Tutor Acadêmico, Universidade A)

Esses serviços contam com a colaboração direta da comunidade, por meio do contato com os Centros de Emprego provinciais, com os projetos direcionados à orientação de estudantes universitários com deficiência, com as associaçóes, as empresas e as organizaçóes. Isso permite que a universidade forneça a todos os estudantes, e não apenas àqueles com deficiências, uma visão ampla das oportunidades e iniciativas promovidas pelo mundo do trabalho, a fim de favorecer que a profissionalizaçáo adquirida durante a carreira acadêmica seja efetivamente colocada em prática na sociedade. 
Além disso, os resultados do estudo evidenciam que, tanto para o serviço de Tutoria Especializada, quanto para o Serviço de Estágios e Colocação no Mercado de Trabalho, o envolvimento dos estudantes, dos professores, da família, dos colegas de classe e dos serviços relacionados, fazendo uso também de ferramentas específicas, é importante nas várias fases do processo de orientação acadêmica e profissional de estudantes com deficiência. Graficamente, podemos representar o funcionamento dos serviços e atores envolvidos diretamente no programa de orientação acadêmica e profissional dos estudantes universitários com deficiência no contexto italiano.

\section{A sinergia entre os vários atores e serviços no programa de orientação acadêmica e profissional na Itália}

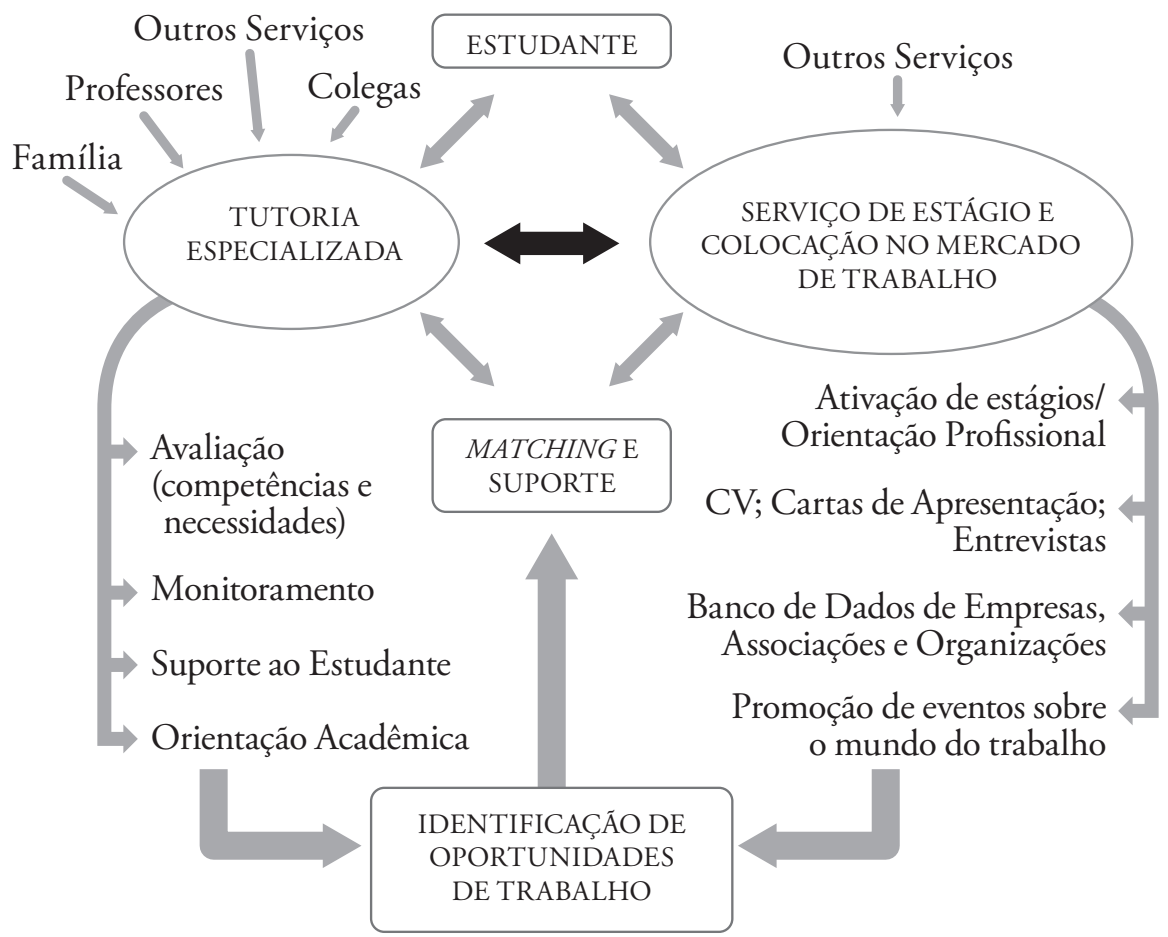

Fonte: Cabral (2013)

Deve-se ressaltar, ainda, que durante a orientação profissional estáo envolvidos o estudante, o sujeito promotor (a universidade) e o anfitriáo, ou seja, a empresa, entidade, associação.

Neste processo, é prevista a presença do tutor universitário (ou um professor da universidade) e o tutor empresarial (não necessariamente o representante legal 
da empresa), que acompanham e orientam o estudante durante o processo de sua transição para o mundo do trabalho. Assim, podemos afirmar que as principais fases de uma orientação acadêmica voltadas à transição do estudante universitário com deficiência ao mundo do trabalho são:

\section{As 9 fases de orientação profissional dos estudantes}

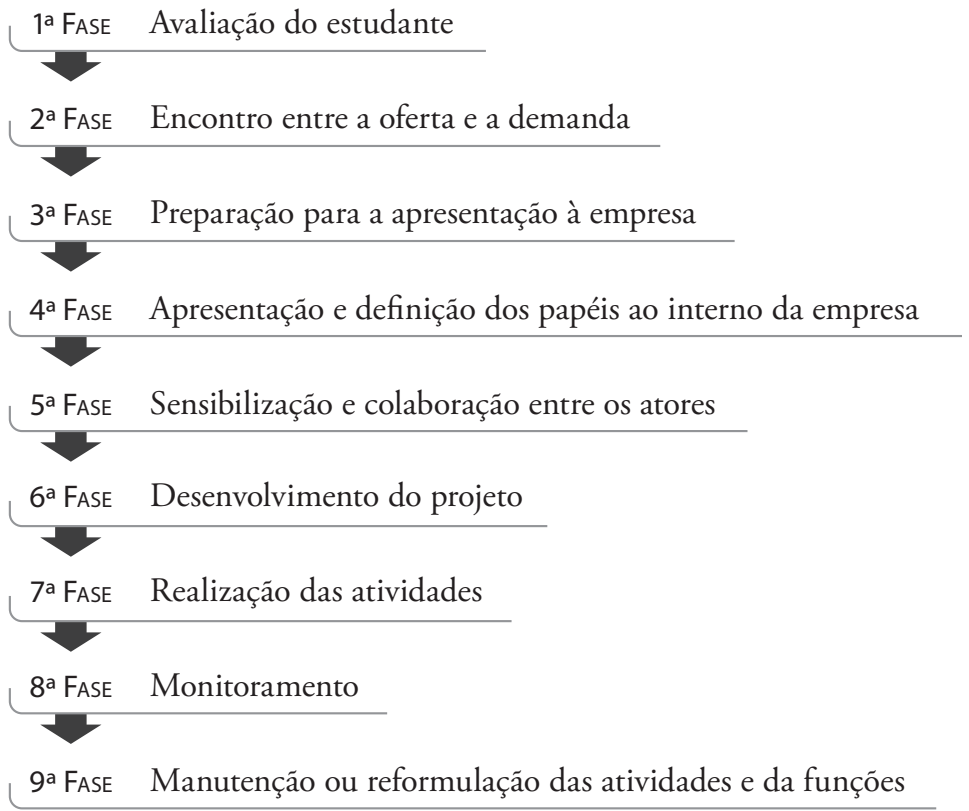

Fonte: Cabral (2013).

Ressalta-se o cuidado de não se implementar um programa de orientação acadêmica e profissional com base em um caráter essencialmente organizacional e tecnicista, mas, sobretudo, tendo em vista os aspectos empíricos individuais, interpessoais, acadêmicos e profissionais dos atores envolvidos no processo de transição para a universidade e para o mundo do trabalho.

Assim, o momento de recepção de um estudante com deficiência, seja na universidade, seja no ambiente de trabalho, é uma das etapas mais importantes do processo de sua orientação acadêmica e profissional. $\mathrm{O}$ tutor acadêmico e o tutor empresarial devem ter, antes de tudo, as habilidades necessárias para poder identificar, em uma abordagem holística, aspectos biopsicossociais e pedagógicos do estudante, sem que eles se sintam em situação de desconforto e, ao mesmo tempo, encorajando-os a uma ativa participação (DE ANNA, 2003, 2005). 


\section{Indicadores para programas de acolhimento e orientação para estudantes com deficiências nas universidades italianas}

Tendo como base as constantes discussóes no grupo de pesquisa, e juntamente com os atores envolvidos no estudo, foi possível identificar indicadores de boas práticas para a orientação acadêmica e profissional dos estudantes universitários com deficiência, os quais foram relatados e socializados, dentre várias ocasióes, a todos os pró-reitores quanto às questôes relacionadas à deficiência da Itália, bem como no Parlamento Europeu de Strasbourg, em 2012, a saber:

1. planejar e desenvolver, desde o início da carreira acadêmica do estudante, uma avaliação inicial e um monitoramento contínuo dos objetivos, necessidades e desenvolvimento do aluno, considerando o seu projeto de vida, o reconhecimento de sua identidade e de suas motivaçôes;

2. promover módulos de formação e de atualização sobre questôes relacionadas e voltadas aos profissionais da universidade e, particularmente, dos serviços de tutoria especializada e de orientação profissional;

3. estabelecer atividades de aconselhamento ao estudante a fim de estimular a assunção de responsabilidade, a solução de problemas que impedem o progresso de seus estudos ou de sua transição para o emprego;

4. promover a relação entre o estudante e o mundo do trabalho por meio das atividades de estágio (pré e pós-graduação) e de formação relacionadas às suas habilidadese e aspiraçóes;

5. incentivar a participação do estudante no programa Sócrates/Erasmus (competências linguísticas) e Leonardo da Vinci (trabalho no exterior);

6. organizar atividades que permitam aos estudantes a aquisição de competências específicas relacionadas à elaboração do Curriculum Vitae e da apresentação pessoal;

7. estabelecer um pareamento eficaz, cruzando a procura e a oferta de trabalho, com base no perfil de cada estudante, à luz dos aspectos acadêmicos, sociais e individuais identificados na avaliação inicial e no monitoramento contínuo;

8. fornecer às empresas eventuais consultorias sobre as competências profissionais adquiridas pelos graduandos e graduados com deficiência e sobre as adequaçóes específicas a serem implementadas no local de trabalho (acessibilidade física, tecnologia, recursos humanos etc.);

9. as universidades devem contactar as estruturas de centros de emprego regionais e os Centros de Orientação Profissional para o trabalho ou qualquer outro serviço responsável por esta questão, de modo a planejar em conjunto e implementar políticas empregatícias por meio da conexáo com a rede de atores públicos e privados que operam no território regional; 
10. instaurar relações com as associaçóes de empresas locais para promover, sempre em colaboraçáo com os serviços de emprego, atividades de sensibilização;

11. promover maiores oportunidades empreendedoras entre os jovens;

12. manter atualizado o banco de dados no qual são organizadas as informaçóes inerentes às oportunidades de estágio e de emprego.

Ao concluir a presente pesquisa, pode-se dizer que um programa de orientação acadêmica e profissional de um estudante com deficiência deve considerar, em uma abordagem holística sobre as habilidades do aluno, o contexto geral da universidade, os serviços de tutoria especializada, de colocaçáo no mercado de trabalho e de estágios, o território e, quando apropriado, a cooperação adequadada da família.

\section{Diagrama representativo dos atores envolvidos no Programa de Orientaçáo Acadêmica e Profissional para os estudantes universitários com deficiência}

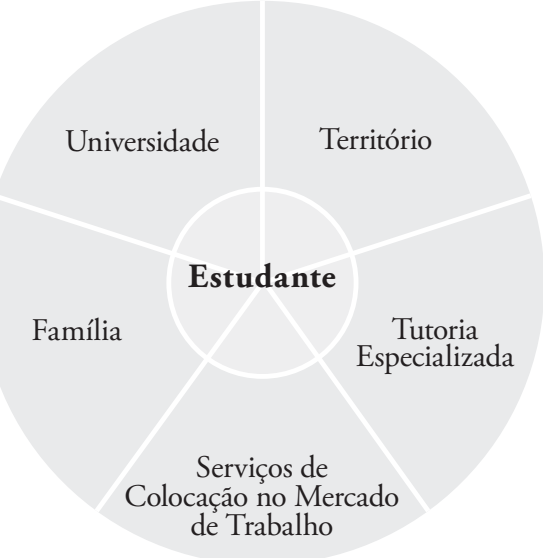

Fonte: Elaboração dos autores.

À luz dos resultados globais deste estudo e de acordo com as indicaçóes da National Alliance for Secondary Education and Transition, de 2005, foi proposto um conjunto de indicadores que poderão auxiliar as diversas estruturas universitárias na implementação e monitoramento de seu próprio programa de orientação acadêmica e profissional de estudantes universitários com deficiência (CABRAL, 2013).

O estudo realizado na Itália, portanto, permitiu-nos não só reconstruir o diálogo internacional sobre o tema desta pesquisa, mas também propor novos instrumentos que dispóem de indicadores para o desenvolvimento de um programa de orientaçáo acadêmica e profissional para a realizaçáo do projeto de vida dos estudantes universitários com deficiência no século XXI. 


\section{Referências}

BROLIN, D.; ELLIOTT, T. R. Meeting the lifelong career development needs of students with handicaps: A community college model. Career Development for Exceptional Individuals, v.7, p.12-21, 1984.

BROWN, J. M.; KAYSER, T. F. Articulation: enhancing special needs students' transitions from secondary to postsecondary vocational education programs. Career Development for Exceptional Individuals, v.4, p.3-7, 1981.

CABRAL, L. S. A. A legislação brasileira e italiana sobre educação especial: da década de 1970 aos dias atuais. 2010. 137f. Dissertação (Mestrado em Educação Especial). Programa de Pós-Graduação em Educação Especial, Universidade Federal de São Carlos, 2010.

CABRAL, L. S. A. Orientação acadêmica e profissional dos estudantes universitários com deficiência: perspectivas internacionais. 226f. 2013. Tese (Doutorado em Educaçáo Especial). Programa de Pós-Graduação em Educação Especial, Universidade Federal de São Carlos, 2013.

CANADÁ. Canadian Council on Rehabilitation and Work (CCRW). Declaração internacional de Montreal sobre inclusão. Trad. Romeu Kazumi Sassaki. In: International Congress Inclusive Society. Montreal, 2001. Disponível em: http://portal.mec.gov.br/seesp/arquivos/pdf/dec_inclu. pdf. Acesso em: 15 maio 2014.

CARTER, E. W; AUSTIN, D; TRAINOR, A. A. Predictors of postschool employment outcomes for young adults with severe disabilities. Journal of Disability Policy Studies, v.23. n.1, p.50-63, 2012.

CNUDD. Conferenza Nazionale Universitaria dei Delegati per la Disabilità. Linee Guida CNUDD. 2001. Disponível em: http://cnudd.di.unipi.it/Documenti\%20condivisi/Forms/ Allitems.aspx. Acesso em: 15 abr. 2014.

COLLINS, B.; O’MAHONY, P. Perceiving success? An investigation of disabled students' and academic staff's perceptions of the factors that hinder a disabled student's occupational performance in Trinity College Dublin. Irish Journal of Occupational Therapy, v.31, n.1, p.1519, 2001.

CONSIGLIO D'EUROPA. Comitato dei Ministri. Piano d'Azione del Consiglio d'Europa 2006-2015 per la promozione dei diritti e della piena partecipazione nella società delle persone con disabilità: migliorare la qualità di vita delle persone con disabilità in Europa. 2006. Disponível em: http://ops.provincia.lecco.it/files/documenti/1182937069.pdf. Acesso em: 10 dez. 2010.

DE ANNA, L. Università e invalidità: un percorso ancora poco agibile. In: Linee del Dipartimento Educazione Scuola dell'Associazione Nazionale Mutilati ed Invalidi Civili (Anmic). Roma: Edizione Tempi Nuovi, 1989.

DE ANNA, L. L'accoglienza degli studenti disabili nelle Università. Roma. In: MINISTERO dell'Università e della Ricerca Scientifica e Tecnologica. Collana Università Ricerca. Notiziario bimestrale, ano VII, p.24-48, mar.-abr. 1996.

DE ANNA, L. Pedagogia speciale: i bisogni educativi speciali. 5. ed. Milano: Guerini Studio, 2002. 197p. (Coleção Processi Formativi e Scienze dell’Educazione).

DE ANNA, L. Alla ricerca del proprio futuro. Universitas, v.89, p. 17-22, set. 2003.

DE ANNA, L. Le esperienze internazionali sull'accoglienza degli studenti Universitari con disabilità. L'integrazione scolastica e sociale, v.4, n.2, p.103-113, 2005.

DE ANNA, L. Pedagogia speciale. Integrazione e inclusione. Roma: Carocci, 2014. 344p.

EBERSOLD, S. La naissance de l'inemployable, ou l'insertion aux risques de l'exclusion. Rennes: Presses Universitaires de Rennes, 2001. 
EBERSOLD, S. Les étudiants handicapés dans l'enseignement supérieur. Paris: OCDE, 2003.

EBERSOLD, S. Disability in higher education: a key factor for improving quality and achievement. 2008a. Disponível em: www.oecd.org/dataoecd/26/49/41887103.ppt. Acesso em: 30 set. 2010.

EBERSOLD, S. L'adaptation de l'enseignement supérieur au handicap : évolution, enjeux et perspectives. In: OCDE (Ed.). L'enseignement supérieur à l'horizon 2030 - Démographie. Paris: OCDE, 2008b. p.241-261.

HOGSTEDT, C.; WEGMAN, D. H.; KJELLSTROM, T. The consequences of economic globalization on working conditions, labor relations and workers' health. In: KAWACHI, I.; WAMAL, S. (Eds.). Globalization and health. Oxford: Oxford University Press, 2007.

KONUR, O. Assessment of disabled student in higher education: current public policy issues. Assessment and Evaluation in Higher Education, v.27, n.2, p.131-152, 2002.

LANDMARK, L. J.; JU, S.; ZHANG, D. Substantiated best practices in transition: fifteen plus years later. Career Development for Exceptional Individuals, v.33. n.3. p.165-176, 2010.

LASCIOLI, A. L'Inserimento lavorativo della persona con disabilità intellettiva. L'integrazione Scolastica e sociale, v.11, n.2, p.114-124, 2012.

MACLEAN, R.; LAI, A. Future of technical and vocational education and training: global challenges and possibilities. International Journal of Training Research, v.9, n.1, p.2-15, 2011.

MENDES, E. G. A radicalização do debate sobre inclusão escolar no Brasil. Revista Brasileira de Educação, Campinas, v.11, n.33, p.387-405, set.-dez. 2006. Disponível em: http://www. scielo.br/pdf/rbedu/v11n33/a02v1133.pdf. Acesso em: 4 maio 2014.

MIUR. Ministero dell'Istruzione, dell’Università e della Ricerca. Atenei. 2012. Disponível em: http://hubmiur.pubblica.istruzione.it/web/universita/home. Acesso em: 13 abr. 2014.

MOLITERNI, P.; DE STASIO, S.; CARBONI, M. Studiare all'Università: strategie di apprendimento e contesti formativi. Milano: Franco Angeli, 2011. 144p. (Collezione Scienze della Formazione).

NORLANDER, K. A.; SHAW, F. M.; MCGUIRE, H. M. Competencies of postsecondary education personnel serving students with learning disabilities. Journal of Learning Disabilities. v.23. p.426-432, 1990.

ONU. Organização das Naçóes Unidas. Declaração dos Direitos das Pessoas Deficientes. Nova York, 1975. Disponível em: http://portal.mec.gov.br/seesp/arquivos/pdf/dec_def.pdf. Acesso em: 15 maio 2011.

ONU. Organização das Naçóes Unidas. Convenzione sui Diritti delle Persone con Disabilità. 2006. Disponível em: http://www.cesq.it/Convenzione_diritti_persone_con_disabilit_int. pdf. Acesso em: 12 set. 2010.

RASKIND, M. H.; HIGGINS, E. L. Assistive technology for postsecondary students with learning disabilities: an overview. Journal of Learning Disabilities, v.31, p.27-40, 1998.

REPETTO, J. B. et al. The High School experience: what students with and without disabilities report as they leave school. Career Development for Exceptional Individuals, v.34, n.3, p.142-152, 2011.

RUSCH, F. R; KOHLER, P. D.; RUBIN, S. Descriptive analysis of OSERS-sponsored postsecondary education model programs. Career Development for Exceptional Individuals, v.17, p.53-63, 1994.

UNESCO. The UN Decade of Education for Sustainable Development: 2005-2014. 2009. 64p. 


\section{Resumo}

Orientaçâo acadêmica e profissional dos estudantes com deficiência nas universidades italianas

O presente artigo é parte de um projeto europeu mais amplo que visou o desenvolvimento de um programa de apoio a estudantes com deficiências durante seu percurso acadêmico e no processo de sua transiçáo ao mercado de trabalho. O objetivo específico do presente estudo consistiu em implementar e avaliar um programa de orientação acadêmica e profissional a esse público alvo. A proposta, baseada num programa já existente no contexto dinamarquês, foi implementada em três universidades italianas, e 43 pessoas foram envolvidas na avaliação de sua eficácia: 20 estudantes universitários com deficiência em fase de transição ao mundo do trabalho, 20 tutores empresariais e 3 tutores acadêmicos. Como resultado, a experiência possibilitou a identificaçáo de elementos-chave relacionados ao percurso acadêmico, à aquisição de competências profissionais dos estudantes, à transiçáo para a vida adulta e o mundo do trabalho. Além disso, foram identificados elementos que contribuem para a constituição do perfil dos tutores acadêmicos e profissionais no ambiente universitário e profissional. Entende-se, com este estudo, que cabe à comunidade científica avançar no processo, reunindo indicadores de boas práticas que possam contribuir para estratégias concretas que garantam a efetiva inclusão social e no trabalho das pessoas com deficiência.

Palavras-chave: Educação Superior. Pessoas com Deficiência. Orientação Acadêmica. Orientação Profissional. Inclusão. Educação Especial.

\section{Abstract}

\section{Academic and Professional Orientation of Students with Disabilities in Italian Universities}

This paper is part of a larger European project that aimed to develop a program for supporting students with disabilities during their academic lives and guide them towards the labor market. The specific objective of this study was to implement and evaluate an academic and vocational guidance program to students with disabilities. Based on a Danish existing program, the proposal was implemented in three Italian universities, and 43 people were involved in the evaluation of its effectiveness: 20 students with disabilities in transition to the labor market, 20 professional tutors and 3 academic tutors. Results showed identification of key factors related to the academic process, the acquisition of students' professional skills, adulthood and labor market transition. Furthermore, elements were identified that helped elaborate a profile of professional and academic tutors both in the university and labor environment. It is understood that the scientific community needs to move within this process, gathering indicators of good practices that can contribute to propose concrete strategies in order to ensure effective social and professional inclusion for people with disabilities.

Keywords: Higher Education. People with Disabilities. Academic Guidance. Vocational Guidance. Inclusion. Special Education. 